\section{Neuroprotektive Ansätze in der Neurologie - Enttäuschte Hoffnungen?}

\author{
F. Zipp ${ }^{1}$ \\ 1 Direktorin der Klinik und Poliklinik für Neurologie, \\ Johannes Gutenberg-Universität Mainz
}

Neuroprotektion ist sicherlich in den verschiedenen neurologischen Erkrankungen unterschiedlich zu bewerten. In der Multiplen Sklerose kann festgehalten werden, dass sich dieses Thema erst in den Anfängen befindet.

Die Multiple Sklerose wird herkömmlich als eine entzündliche, demyelinisierende Erkrankung des zentralen Nervensystems gesehen, in der die schubförmigen remittierenden neurologischen Symptome aus der Beschädigung der Myelinscheide, d.h. Entmarkung oder Demyelinisierung, entstehen. Multiple Sklerose ist die häufigste chronisch entzündliche Erkrankung des Zentralnervensystems in westlichen Ländern und führt zu einschneidenden Behinderungen bei jungen Leuten, für die die bislang zur Verfügung stehenden therapeutischen Möglichkeiten immer noch nicht zufriedenstellend sind. Sozioökonomisch stellt diese Erkrankung eine große Aufgabe dar. Junge Leute erkranken in der Regel in einem Lebensabschnitt, in dem wesentliche Entscheidungen zu Beruf und Familie anstehen. Es finden sich komplexe Bilder in der Pathologie mit disseminierter Entzündung (mit Entmarkung, Remyelinisierung, Nervenfaser-Schädigung) subkortikal und kortikal sowie Neurodegeneration. Remissionen nach klinischen Schüben deuten auf eine Reparatur-Kapazität des Zentralnervensystems hin, die sehr unterschiedlich sein kann von Patient zu Patient und im Verlauf der Erkrankung. Trotz intensiver Forschung auf dem Gebiet in den letzten drei Jahrzehnten sind die zugrundeliegenden molekularen Mechanismen nach wie vor nicht geklärt und es kann im Wesentlichen keine prognostische Einschätzung des Verlaufs erfolgen. Immunmodulatorische und -suppressive Therapien wurden parallel zu anderen Autoimmunkrankheiten entwickelt. Jedoch unterscheidet sich die Multiple Sklerose diesbezüglich von anderen Autoimmunkrankheiten. Die zur Verfügung stehenden Therapien haben wenig Einfluss auf die Krankheits- und Behinderungsprogression. Zusätzlich sind die neuen Therapien in der Multiplen Sklerose mit deutlichen Risiken einer opportunistischen Krankheit assoziiert. Daher muss therapeutisch noch einmal grundsätzlich an neuen Strategien gearbeitet werden.

In den letzten zehn Jahren ist klar geworden, dass neurodegenerative Mechanismen auch eine wichtige Rolle in der Pathologie der Multiplen Sklerose spielen. Moderne histopathologische und zunehmend auch Bildgebungsmethoden können eingesetzt werden, um die wesentlichen neurologischen Schäden in Form von axonaler Pathologie und früh vorkommendem neuronalen Verlust sowie transiente neuronale
Schädigung experimentell und in Ansätzen auch klinisch nachzuweisen. Diese Schäden sind besonders ausgeprägt in den Demyelinisierungsherden, ganz gleich, in welcher Phase der Erkrankung sie auftreten. Die axonale Pathologie, die sich aus der Schädigung der isolierenden Funktion der Myelinscheide ergibt, ist somit am ehesten der verantwortliche Pathomechanismus in schwer demyelinisierten Regionen. Die veränderte Verteilung und Expression verschiedener Ionenkanäle und Transporter führt hier zu einer örtlichen Akkumulation intrazellulären Calciums in den demyelinisierten Neuronen und damit zu dem darauffolgenden neuronalen Zelltod. Diese Mechanismen sind noch nicht voll verstanden. Sie scheinen ebenso eine Rolle zu spielen, wenn Neurone direkt von T-Lymphozyten angegriffen werden, vermutlich in einem frühen Krankheitsstadium. Hier kommt die in histopathologischen Untersuchungen festgestellte vermehrte neuronale Pathologie nämlich nicht nur in den typischen Demyelinisierungsherden, sondern auch in morphologisch scheinbar normaler grauer und weißer Substanz (normal-appearing gray and white matter) vor. Diese Schädigung ist damit möglicherweise unabhängig von der entzündlichen Demyelinisierung.

Die Schädigung von neuronalen Strukturen hat, wie wir aus der Kernspintomografie wissen, eine viel größere Bedeutung für die tatsächliche Behinderung der jungen Patienten, als dies die Einwanderung der Entzündungszellen mit Angriff zunächst auf die Markscheiden hat. Es gibt mittlerweile zwei Arbeitsgruppen, die gezeigt haben, dass die erste Phase der Erkrankung bis zu einer sichtbaren Behinderung offensichtlich sehr variabel hinsichtlich der Schübe und der zeitlichen Ausdehnung verlaufen kann. Wenn eine sichtbare Behinderung (EDSS 3-4) erreicht ist, dann scheint die Progression der Behinderung sehr stereotyp zu verlaufen unabhängig von Schüben. Auf der Basis dieser Beobachtungen, kernspintomographischer Untersuchungen und experimenteller Hinweise muss man davon ausgehen, dass neuroprotektive Therapien am besten früh eingesetzt werden sollten und mit antientzündlichen Therapieansätzen synergistische Wirkung entfalten könnten. Zu einem späteren Zeitpunkt in der Erkrankung sind möglicherweise nur noch Medikamente mit Reparaturpotenzial sinnvoll. Ob wir an dieser Stelle noch etwas mit neuroprotektiven Strategien erreichen können, werden klinische Therapie-Studien in den nächsten Jahren zeigen.

Die bislang wenigen neuroprotektiven und neuroregenerativen Studien, die an Patienten mit Multipler Sklerose durchgeführt wurden, sind methodologisch sicherlich nicht ausreichend gut konzipiert gewesen, um klar Wirkprinzipien zu beoder widerlegen. Die neuen Therapien, die sich in der späten Phase III oder im Zulassungsverfahren befinden, wie z.B. Alemtuzumab, Sphingosin-Phosphat-Rezeptor-Modulatoren oder Fumurat, weisen hauptsächlich antientzündliche Wirkmechanismen auf. Experimentelle, klinische und Bildgebungsdaten deuten jedoch darauf hin, dass sowohl bereits zugelassene als auch die neu entwickelten Substanzen potentiell auch protektive oder möglicherweise auch reparative Eigenschaften haben könnten, die über eine reine Entzün- 
dungshemmung hinausgehen. Durch die Untersuchung dieser Wirkmechanismen und ebenso durch unsere experimentellen und klinischen Untersuchungen können wir möglicherweise ganz neue Erkenntnisse zur Pathologie und damit besseren Behandlung dieser chronisch-autoimmunen Erkrankung, den Schädigungsmechanismen und ihrer Blockade sowie Reparaturmöglichkeiten gewinnen.
Interessenkonflikt: Die Autorin erklärt, dass sie in keinem Interessenkonflikt steht.

Bibliografie

Dol http://dx.doi.org/10.1055/s-0033-1346711

Drug Res 2013; 63, Suppl. 1: S12-S13

(c) Georg Thieme Verlag KG Stuttgart · New York .

ISSN 2194-9379 